\title{
Efecto del coste percibido, la calidad de servicio y la satisfacción sobre las intenciones futuras del espectador
}

\author{
FERRAN CALABUIG MORENO
}

Departamento de Educación Física y Deportiva, UNIVERSIDAD DE VALENCIA, ESPAÑA. E-mail: ferran.calabuig@uv.es

JOSEP CRESPO HERVÁS

Departamento de Educación Física y Deportiva, UNIVERSIDAD DE VALENCIA, ESPAÑA. E-mail: josep.crespo@uv.es

\section{JAVIER MUNDINA GÓMEZ}

Departamento de Educación Física y Deportiva, UNIVERSIDAD DE VALENCIA, ESPAÑA. E-mail: jose.j.mundina@uv.es

\section{RESUMEN}

Recientemente se ha introducido el estudio del valor como una variable que interviene en la formación de la satisfacción y las intenciones de recompra. Este trabajo analiza la calidad percibida, el valor coste percibido, la satisfacción y las intenciones futuras de los espectadores de un gran evento deportivo. Mediante modelos de ecuaciones estructurales se encuentra que hay un fuerte efecto del valor coste sobre la satisfacción. Además, se observa que el valor es la variable que mayor peso tienen en la predicción de las intenciones futuras de los espectadores.

Palabras clave: Eventos deportivos, valor monetario, valor percibido, calidad de servicio, satisfacción.

\section{Effect of Perceived Cost, Service Quality and Satisfaction on Future Intentions of Spectators}

\begin{abstract}
Recently the study of perceived value has been introduced as a variable that has a strong influence in the development of satisfaction and the repurchase intention. This work provides an analysis of the perceived quality, value, satisfaction and future intentions of spectators in a mega sports event. Trough structural equations modeling results indicate a strong effect of cost value on satisfaction. It is further noted that cost value is the variable that has more weight in predicting the future intentions of spectators.
\end{abstract}

Keywords: Sporting Events, Cost Value, Perceived Value, Service Quality, Satisfaction.

Clasificación JEL: M31

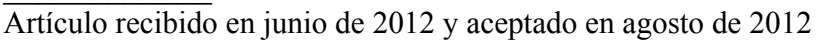

Artículo disponible en versión electrónica en la página www.revista-eea.net, ref. ə-30220 


\section{INTRODUCCIÓN}

El análisis de los eventos deportivos es un tema recurrente en la investigación sobre economía y gestión del deporte ya sea para conocer el impacto económico sobre las ciudades sede, para crear modelos predictivos de las victorias de los equipos, para conocer la estructura de premios en las quinielas, para determinar el valor de los jugadores o equipos, para conocer la influencia del balance competitivo en la asistencia de espectadores a los estadios o cuáles son los factores que más influyen en la satisfacción o en la intención de repetir de los espectadores, entre otros (Calabuig et al., 2012).

Desde esta última perspectiva y entendiendo los eventos deportivos como un servicio, la relación que se establece entre la calidad de servicio, la satisfacción, el valor percibido y las intenciones futuras es un tema recurrente en la literatura de los servicios en general (Cronin et al., 2000) y de la gestión del deporte en particular (Kwon, Trail y Anderson, 2005). Este interés se ha trasladado al estudio de la calidad de servicio y la satisfacción de los usuarios en centros deportivos (Tsitskari et al., 2006) y se está extendiendo a los eventos deportivos (Bodet y Bernanche-Assollant, 2011). En el caso de los eventos deportivos hay que destacar en España, el Eventqual de Calabuig et al. (2010b) y en el ámbito internacional la aportación de Ko et al. (2011).

Todo este interés viene motivado por la influencia positiva que tiene la satisfacción de los consumidores de servicios con los beneficios a medio plazo que tiene el hablar bien de la empresa, la venta cruzada (cross-buying) o la lealtad del cliente (Anderson et al., 1994). Es conocido que la satisfacción es un elemento clave en las estrategias de retención y fidelización de los clientes (Cronin et al., 2000) y esto es lo que la convierte en un atractivo para los gestores del deporte e investigadores. En los eventos deportivos, se ha encontrado que la satisfacción del espectador es un predictor o antecedente significativo de las intenciones futuras de asistir (Cronin et al., 2000; Kim et al., 2006; Wakefield y Blodgett, 1996) y que a su vez la satisfacción es una consecuencia del valor percibido (Calabuig et al., 2010b; Cronin et al., 2000) y de la calidad (Calabuig et al., 2010b; Tsuji et al., 2007).

En consecuencia, en este trabajo se plantea un modelo estructural entre las variables mencionadas para intentar desgranar las relaciones que existen entre la calidad percibida, el valor coste percibido, la satisfacción y las intenciones futuras del espectador, el cual se plasma en la Figura 1. Como se puede observar en la figura, se obvian los ítems (variables observables) que conforman cada factor (variables no observables) con el objetivo de facilitar la interpretación y visualización de las relaciones del modelo. Asimismo, se indican las hipótesis a contrastar en cada relación, que en el siguiente capítulo se fundamentan y explican. 
Figura 1

Variables introducidas en el modelo estructural, relaciones e hipótesis

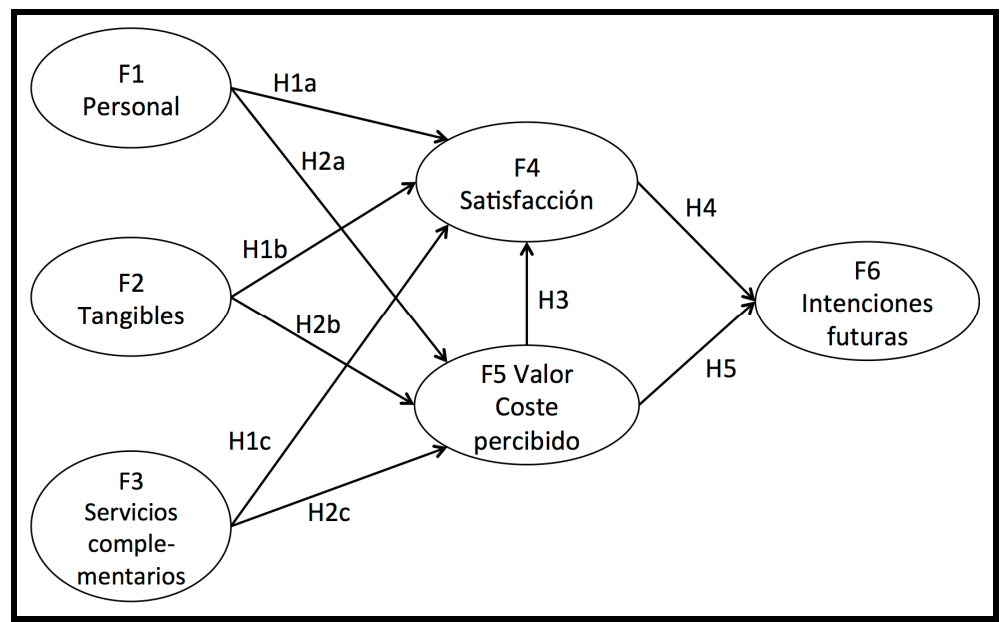

Fuente: Elaboración propia.

Según la notación del programa EQS 6.1, y a partir del diseño propuesto en la Figura 1, el modelo de ecuaciones estructurales que se pone a prueba queda de la siguiente manera:

$$
\begin{aligned}
& \mathrm{F} 4=* \mathrm{~F} 5+* \mathrm{~F} 1+* \mathrm{~F} 2+* \mathrm{~F} 3+\mathrm{D} 4 \\
& \mathrm{~F} 5=* \mathrm{~F} 1+* \mathrm{~F} 2+* \mathrm{~F} 3+\mathrm{D} 5 \\
& \mathrm{~F} 6=* \mathrm{~F} 4+* \mathrm{~F} 5+\mathrm{D} 6
\end{aligned}
$$

Dónde F es la variable latente o no observable

* es el coeficiente de regresión y,

$D$ es el término de error

Teniendo en cuenta todo esto, los objetivos que se plantean en este estudio son: a) testar un modelo de relaciones causales en el que la satisfacción y el valor coste percibido son función de la calidad percibida, y que las intenciones futuras son función de la satisfacción y el valor coste percibido y b) conocer la importancia del valor coste en la predicción de las intenciones futuras del espectador.

\section{REVISIÓN DE LA LITERATURA E HIPÓTESIS}

\subsection{Calidad percibida y satisfacción del espectador}

La calidad de servicio ha sido definida como una actitud duradera que se expresa como la diferencia entre las expectativas y el resultado del servicio (Parasuraman et al., 1988). Un inconveniente de esta perspectiva es que las 
expectativas son ciertamente difíciles de detectar y medir. Por ello, Cronin y Taylor (1992) también aceptaron que la calidad de servicio percibida es la diferencia entre las expectativas y el resultado del servicio, pero entendían que dicha diferencia ya la realiza el consumidor cuando es preguntado por el resultado final del servicio, y por tanto proponen medir únicamente la percepción y no considerar las expectativas.

Por otra parte, la satisfacción puede ser definida como la comparación entre las expectativas y la percepción de la calidad del servicio recibido por el usuario (Shonk y Chelladurai, 2008). Aunque, desde un punto de vista más hedónico, también es entendida como una reacción placentera hacia un bien o servicio en un acto de consumo (Oliver, 1997). Esta es más volátil y efímera y además es más fácil de ser modificada con cada acto de consumo. En cambio, la calidad percibida es considerada como más estable, duradera en el tiempo y difícil de cambiar.

Diversos estudios en el ámbito de la gestión del deporte encuentran que la calidad de servicio es un predictor de la satisfacción (Greenwell et al., 2002; Tsuji et al., 2007). En este sentido Theodorakis et al. (2001) analizan la calidad percibida de los espectadores de baloncesto y determinan que las dimensiones que mayor peso tienen en la predicción de la satisfacción de los espectadores son la fiabilidad y los tangibles. En este caso, la fiabilidad está formada por ítems referidos a la habilidad para ejecutar el servicio prometido de forma fiable y cuidadosa, y los tangibles contiene ítems relacionados con la apariencia de las instalaciones físicas, equipos, personal y materiales de comunicación. En cambio, Kelley y Turley (2001) observan que el atributo más importante es el relacionado con el espectáculo deportivo, aunque asumen que no es susceptible de modificación por parte de los gestores. Greenwell et al. (2002) encuentran que los tangibles influyen en la satisfacción de los espectadores de hockey hielo de un modo global más que evaluándolos como elementos individuales (comodidad, accesos,...). Así, las hipótesis que se plantean son:

H1a. La percepción del espectador respecto de la calidad del personal del evento tiene una relación positiva y significativa con su satisfacción.

H1b. La percepción de calidad de los elementos tangibles del evento tienen una relación positiva y significativa con la satisfacción del espectador.

H1c. La calidad de los servicios complementarios del evento tiene una relación positiva y significativa con la satisfacción del espectador.

\subsection{Valor percibido, satisfacción e intenciones futuras del espectador}

Zeithaml (1988, p.14) define el valor percibido como "la valoración global del consumidor de la utilidad de un producto basado en la percepción de lo que es recibido y de lo que es dado". En esta línea, Chen y Dubinsky (2003) indican 
que el valor percibido del consumidor es la percepción que hace del conjunto de beneficios obtenidos en el intercambio y el coste incurrido para obtener los beneficios deseados. Encontramos investigadores que consideran que el beneficio es sinónimo de calidad, y sacrificio es identificado con precio. Dodds et al. (1991) conceptualizaron el valor percibido como una compensación entre la calidad percibida y el sacrificio monetario. Es entonces cuando hablamos de valor coste percibido.

Existen distintos trabajos que ponen de manifiesto que la calidad del servicio es un antecedente del valor percibido (Chen y Dubinsky, 2003; Hume, 2008). También encontramos algún trabajo en eventos deportivos que mantienen esta relación (Cronin et al., 2000) aunque la investigación en este ámbito ha sido muy escasa. A este respecto las hipótesis que se plantean son:

H2a. La calidad del personal tiene una relación directa y positiva sobre el valor coste percibido.

$\mathrm{H} 2 \mathrm{~b}$. Los elementos tangibles del evento tienen una relación directa y positiva sobre el valor coste percibido.

H2c. Los servicios complementarios del evento tienen una relación directa y positiva sobre el valor coste percibido.

Por otra parte muchos estudios consideran que la satisfacción es una consecuencia del valor percibido (Sánchez e Iniesta, 2006; Setijono y Dahlgaard, 2007), como se pone de manifiesto en la literatura en la que se da esta relación (Caruana y Fenech, 2005; Cronin et al., 2000; Gallarza y Gil, 2006). En los eventos deportivos Calabuig et al. (2010a), Cronin et al. (2000) y Hightower et al. (2002) encuentran que el valor es un fuerte predictor de la satisfacción del espectador. La hipótesis que se propone es:

H3. El valor coste percibido por el espectador tiene una relación positiva y directa sobre su satisfacción general.

Respecto a la relación de la lealtad con la satisfacción Chang et al. (2009) manifiestan que la satisfacción afecta directamente a la lealtad del consumidor, la cual engloba dimensiones como intención de recompra, boca-oído y la tolerancia a un incremento de precios (Anderson et al., 1994; Fornell et al., 1996). En la literatura se reconoce mediante diversas publicaciones la influencia positiva de la satisfacción sobre la intención (Cronin y Taylor, 1992; Fornell, 1992; Price y Arnould, 1999; Wangenheim y Bayón, 2007) algunas de ellas centradas específicamente en el ámbito de los espectáculos deportivos (Wakefield y Blodgett, 1996; Cronin et al., 2000; Laverie y Arnett, 2000; Trail et al., 2005; Kim et al., 2006). Por tanto, la hipótesis a contrastar es:

H4. Existe una relación directa y positiva entre la satisfacción y las intenciones futuras del espectador. 
Al considerarse el valor percibido como una medida de valoración global del consumidor sobre la utilidad de un servicio o producto basado en las percepciones de lo que es recibido y lo que es dado (Zeithaml, 1988), parece evidente que la lealtad venga determinada por el valor percibido. De este modo, varios estudios sugieren que el valor percibido es uno de los determinantes más importantes de la intención de recompra y de la intención de revisita (Bojanic, 1996; Jayanti y Ghosh, 1996). En el ámbito de los eventos deportivos destaca el trabajo de Clemes et al. (2011), los cuales confirman esta relación. Así pues, la hipótesis que se plantea es:

H5. Existe una relación directa y positiva entre el valor coste percibido y las intenciones futuras del espectador.

\section{MATERIAL Y MÉTODOS}

\subsection{Muestra}

La investigación se desarrolló durante la celebración de un mega-evento deportivo. Se administró un cuestionario sobre calidad de servicio percibida, valor coste percibido, satisfacción e intenciones futuras a los espectadores recogiendo 1.104 cuestionarios válidos. La muestra se compone por un 58,8\% de hombres y un $41,2 \%$ de mujeres con una edad media de 32,97 años $( \pm 11,3)$. El 39\% de los encuestados tiene 36 o más años, el 37,8\% tiene entre 24 y 35 años. El 12,2\% tiene entre 19 y 23 años y el $11 \%$ entre 16 y 18 años.

\subsection{Instrumentos}

La escala utilizada para el análisis de la calidad percibida original se componía de 18 ítems con una alternativa de respuesta de siete puntos que oscilaba entre totalmente en desacuerdo (1) y totalmente de acuerdo (7) de Calabuig et al. (2010b). Esta escala se modificó y se reespecificó pues el análisis factorial confirmatorio (AFC) mediante modelos de ecuaciones estructurales (SEM) sugirió eliminar 5 ítems, reduciéndose la escala en una dimensión quedando así en 3 dimensiones y 13 indicadores. Las tres dimensiones de calidad resultantes son el personal, los tangibles y los servicios complementarios. Para conocer la satisfacción general se administró la escala de Hightower et al. (2002) compuesta por 4 ítems con una alternativa de respuesta de siete puntos. El valor coste percibido se determinó mediante dos ítems con una alternativa de respuesta de 7 puntos. Éstos ítems son "en general asistir al evento compensa lo pagado" y "al asistir al evento he recibido mucho por el dinero que he pagado". Las intenciones futuras se evaluaron a través de tres ítems de Zeithmal et al. (1996) con una alternativa de respuesta de 7 puntos. Los indicadores fueron "estoy dispuesto a continuar asistiendo al evento", "recomendaré la asistencia al evento a amigos y familiares" y " si tengo la oportunidad de volver, repetiré la experiencia". 


\subsection{Análisis de datos}

El análisis estadístico de los datos se realizó mediante el paquete estadístico SPSS 18 y el programa EQS 6.1. En primer lugar se procedió al cálculo de los estadísticos descriptivos para cada ítem, tras lo cual se procedió a evaluar la fiabilidad y validez de la escala. Finalmente se realizó una análisis factorial confirmatorio el modelo de relaciones estructurales mediante el programa EQS. En el análisis SEM se partió de la matriz de datos original.

\section{RESULTADOS}

En este capítulo, se examinará primeramente la fiabilidad y validez de las escalas de medida utilizadas en el investigación y posteriormente se analizarán las relaciones causales en el modelo propuesto.

\subsection{Análisis de las escalas: fiabilidad y validez}

\subsubsection{Fiabilidad de las escalas de medida}

El análisis de las escalas utilizadas para medir los constructos del modelo se divide en dos tramos, en el primero de ellos se analiza la fiabilidad individual para posteriormente proceder a su análisis conjunto, comprobando de esta manera su validez y fiabilidad de concepto. Para el análisis de la fiabilidad se utilizará: a) el coeficiente de alpha de Cronbach siguiendo las recomendaciones de Nunnally y Bernstein (1994). b) el coeficiente de fiabilidad compuesta para cada una de las dimensiones críticas, y c) la varianza media extraída.

Todos los valores del alpha de Cronbach fueron superiores a 0,7. A continuación se procede a aplicar un análisis factorial confirmatorio (AFC) para un examen más exhaustivo siguiendo las recomendaciones de Bagozzi y Yi, (1988).

El coeficiente de fiabilidad compuesta se considera más adecuado que el alfa de Cronbach porque no depende del número de atributos asociados a cada concepto (Vandenbosch, 1996). Todos los coeficientes mostraron un valor superior a 0,7 , valor mínimo recomendado (Bagozzi y Yi, 1988) tanto para la fiabilidad compuesta como para la fiabilidad individual de los ítems. En la Tabla 1 se resumen estos resultados.

Respecto a la varianza media extraída (AVE) (Fornell y Larcker, 1981), refleja la cantidad total de la varianza de los indicadores recogida por el constructo latente. Cuanto mayores sean los valores de AVE más representativos son los indicadores de la variable latente en la que cargan, se recomienda que dichos valores sean superiores a 0,50 (Bagozzi y Yi, 1988; Hair et al., 2006). En la Tabla 1 se puede observar cómo todos los constructos superan este punto de corte. No obstante, aunque la dimensión de tangibles se encuentra ligeramente 
por debajo del 0,50 recomendado, no es impedimento para confirmar la fiabilidad de los instrumentos tomando en consideración el resto de indicadores.

Tabla 1

Medidas de fiabilidad de las escalas utilizadas

\begin{tabular}{|l|c|c|c|}
\hline \multicolumn{1}{|c|}{ Concepto } & $\begin{array}{c}\text { Alpha de } \\
\text { Cronbach }\end{array}$ & $\begin{array}{c}\text { Fiabilidad } \\
\text { compuesta }\end{array}$ & AVE \\
\hline Satisfacción & 0,88 & 0,90 & 0,70 \\
\hline Valor Coste & 0,72 & 0,70 & 0,53 \\
\hline Intención Futura & 0,87 & 0,87 & 0,69 \\
\hline Personal & 0,84 & 0,85 & 0,58 \\
\hline Tangibles & 0,79 & 0,84 & 0,48 \\
\hline Servicios complementarios & 0,75 & 0,77 & 0,54 \\
\hline
\end{tabular}

Fuente: Elaboración propia.

\subsubsection{Validez de las escalas de medida}

En esta investigación se ha analizado la validez de las escalas a través de la validez convergente y la validez discriminante. La validez convergente puede comprobarse analizando las correlaciones entre las diferentes dimensiones que integran el constructo sobre el que se está trabajando (Gómez y Palací, 2003). Dado que se analiza un constructo, las dimensiones que lo definen deben de estar correlacionadas entre sí de forma significativa.

Así pues, analizadas las cargas estandarizadas de los elementos, se observa como todas ellas fueron significativas en sus respectivas dimensiones $(p<0,05$; $\mathrm{t}$ valor > 5) y, del mismo modo, las correlaciones entre pares de constructos también fueron significativas. En consecuencia se puede afirmar validez convergente (Anderson y Gerbing, 1988).

A continuación se procedió a comprobar la validez discriminante. Para ello, se analizaron las correlaciones entre los factores y los intervalos de confianza. Las correlaciones al cuadrado entre pares de factores resultaron menores que la varianza extraída para ese constructo (Fornell y Larcker, 1981). Por otra parte, las correlaciones entre pares de constructos no fueron excesivamente altas $(<$ $0,85)$, lo que también indica la existencia de validez discriminante (Luque-Martínez, 2004). Se recurrió por último al test del intervalo de confianza (Anderson y Gerbing, 1988). La condición que dentro de los intervalos de \pm 2 errores estándar entre la correlación entre los factores no se encuentre el valor 1, se cumple en el presente trabajo. Por tanto, se puede asumir validez discriminante. 


\subsubsection{Ajuste del modelo factorial confirmatorio}

Mediante SEM se procedió a confirmar la estructura factorial reespecificada de la escala de calidad percibida, la cual está formada por 3 dimensiones y 13 indicadores o ítems. Se puede consultar la estructura factorial en el anexo.

Varios índices de ajuste fueron la base para evaluar el modelo: Goodness of Fit Index (GFI) (Jöreskog y Sörbom, 1986), Comparative Fit Index (CFI) (Bentler, 1990). Además, se incluyen otros índices como la chi cuadrado y el Root Mean Square Error of Approximation (RMSEA) (Steiger, 1990) que se consideran apropiados para la evaluación del modelo (Hu and Bentler, 1999). Los resultados indican un adecuado ajuste $(\chi 2: 741,32$, df: 222 , p $<0,001$; GFI: 0,90; y CFI: 0,93; RMSEA: 0,058).

\subsection{Análisis de las relaciones causales}

Una vez comprobadas la fiabilidad y validez de las escalas empleadas, se procede al análisis de las relaciones causales del modelo (Kaplan, 2000). Para tal propósito se utilizó el programa informático EQS 6.1 (Bentler, 1995). Primero se muestran los indicadores de ajuste del modelo propuesto y posteriormente se analizan las relaciones causales. En la Tabla 2 se puede encontrar un resumen de los resultados arrojados por la modelización.

\subsubsection{Ajuste del modelo estructural}

El proceso de estimación del modelo se hizo mediante máxima verosimilitud con la corrección robusta de Satorra-Bentler (Bentler, 1995). Dado que el estadístico Chi-cuadrado $(\chi 2)$ es muy susceptible a tamaños muestrales grandes (Sánchez, 1996) se procedió a examinar otros indicadores, como el ratio entre Chi-cuadrado y sus grados de libertad, siendo aceptables los valores inferiores a cinco (Byrne, 1989; Carmines y McIver, 1981), índices de bondad de ajuste como el Normed Fit Index (NFI), el Non-Normed Fit Index (NNFI), el Comparative Fit Index (CFI) y el Incremental Fit Index (IFI), siendo indicadores de un buen ajuste los valores superiores a 0,90 (Maccallum y Austin, 2000). Asimismo, también se analizó el Root Mean-Square Error of Approximation (RMSEA), pudiéndose aceptar como indicador de un ajuste adecuado las puntuaciones menores a 0,08 (Browne y Cudeck, 1993).

El modelo estructural mostró buenos índices de bondad de ajuste (S-B $\chi 2$ : 467,36, gl: 197, p<0,001; S-B $\chi 2 / g 1: ~ 2,37$; NFI: 0,90; NNFI: 0,91; CFI: 0,92; IFI: 0,92; RMSEA: $0,049(0,044-0,055))$.

\subsubsection{Análisis de las relaciones estructurales}

Respecto a las relaciones causales del modelo (ver Figura 2), se puede decir en primer lugar, que se confirma la hipótesis 1, es decir, existe una influencia 
directa y positiva que ejercen las dimensiones de calidad sobre la satisfacción: personal (H1a: $\lambda=0,182 ; \mathrm{p}<0,05)$, tangibles (H1b: $\lambda=0,317 ; \mathrm{p}<0,05)$ y servicios complementarios (H1c: $\lambda=0,028 ; \mathrm{p}<0,05)$ (Oliver, 1997; Szymanski y Henard, 2001).

Tabla 2

Coeficientes y medidas de ajuste del modelo

\begin{tabular}{|c|c|c|c|}
\hline Hipótesis & Relación estructural planteada & $\begin{array}{c}\text { Estimador } \\
\text { estandarizado }(\lambda)\end{array}$ & Resultado \\
\hline $\mathrm{H}_{1 \mathrm{a}}$ & Personal $\rightarrow$ Satisfacción & 0,182 & Aceptada \\
\hline $\mathrm{H}_{1 \mathrm{~b}}$ & Tangibles $\rightarrow$ Satisfacción & 0,317 & Aceptada \\
\hline $\mathrm{H}_{1 \mathrm{c}}$ & Serv. complementarios $\rightarrow$ Satisfacción & 0,028 & Aceptada \\
\hline $\mathrm{H}_{2 \mathrm{a}}$ & Personal $\rightarrow$ Valor Coste & 0,189 & Aceptada \\
\hline $\mathrm{H}_{2 \mathrm{~b}}$ & Tangibles $\rightarrow$ Valor Coste & 0,541 & Aceptada \\
\hline $\mathrm{H}_{2 \mathrm{c}}$ & Serv. Complementarios $\rightarrow$ Valor Coste & 0,031 & Aceptada \\
\hline $\mathrm{H}_{3}$ & Valor Coste $\rightarrow$ Satisfacción & 0,417 & Aceptada \\
\hline $\mathrm{H}_{4}$ & Satisfacción $\rightarrow$ Intención Futura & 0,046 & Aceptada \\
\hline $\mathrm{H}_{5}$ & Valor Coste $\rightarrow$ Intención Futura & 0,855 & Aceptada \\
\hline
\end{tabular}

Fuente: Elaboración propia.

La hipótesis 2 ponía a prueba la relación de la calidad con el valor coste percibido. Se ha constatado que las dimensiones de personal (H2a: $\lambda=0,189$; $\mathrm{p}<0,05)$, tangibles $(\mathrm{H} 2 \mathrm{~b}: \lambda=0,541 ; \mathrm{p}<0,05)$ y servicios complementarios $(\mathrm{H} 2 \mathrm{c}$ : $\lambda=0,031 ; \mathrm{p}<0,05)$, tienen un efecto significativo y positivo sobre el valor. Por tanto, la calidad también ejerce una influencia directa sobre el valor percibido (Clemes et al., 2011).

También se confirma la tercera hipótesis, es decir, el valor coste percibido ejerce un efecto positivo y significativo sobre la satisfacción (H3: $\lambda=0,417$; $\mathrm{p}<0,05)$. Del mismo modo, ha resultado en el sentido teórico esperado la relación entre la satisfacción y la intención $(\mathrm{H} 4: \lambda=0,046 ; \mathrm{p}<0,05)$ tal y como se había señalado en la literatura especializada (Baker y Crompton, 2000; Oliver et al., 1997) aunque en este caso con una fuerza escasa.

Por último, la hipótesis 5 testaba la relación entre el valor coste percibido y las intenciones futuras del espectador. En este sentido, es destacable el fuerte efecto que ejercer el valor coste sobre las intenciones futuras del espectador (H5: $\lambda=0,855 ; \mathrm{p}<0,05)$.

Finalmente, en la Figura 2 se puede observar como las relaciones planteadas explican un porcentaje moderadamente elevado de la varianza de las variables dependientes, en este caso la satisfacción $\left(\mathrm{R}^{2}=0,664\right)$, el valor coste percibido $\left(\mathrm{R}^{2}=0,479\right)$ y las intenciones futuras $\left(\mathrm{R}^{2}=0,674\right)$. 
Figura 2

Modelo de relaciones estructurales estimado

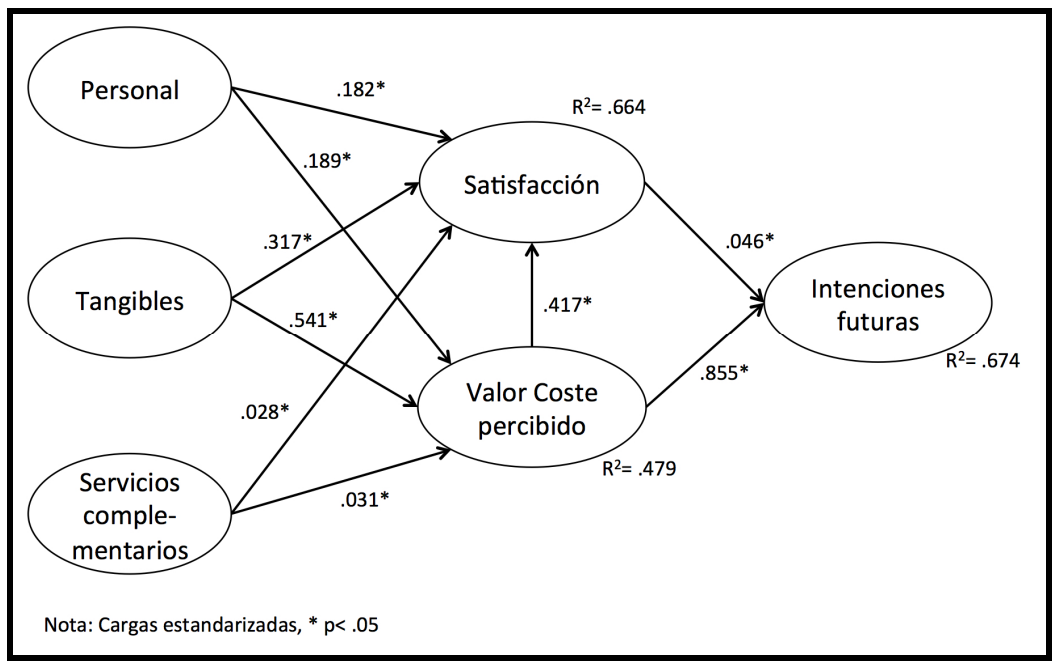

Fuente: Elaboración propia.

\section{DISCUSIÓN Y CONCLUSIONES}

En relación al primer objetivo, los resultados indican que el modelo estructural propuesto es soportado empíricamente con buenos indicadores de ajuste y de fiabilidad y validez de las medidas. Respecto a la calidad, los espectadores perciben tres dimensiones. Estas son el personal, los tangibles y los servicios complementarios. En este estudio se ha descartado la dimensión de accesibilidad pues los indicadores de fiabilidad, validez y ajuste del modelo con ella incluida no eran los esperados. La accesibilidad es una de las dimensiones que suele estar presente en los eventos deportivos (Calabuig, et al., 2010b; Clemes et al., 2011; Kelley y Turley, 2001; Theodorakis y Alexandris, 2008) pero en este caso se puede indicar que en los eventos donde los espectadores son buenos conocedores del servicio no les resulta importante, o al menos no es achacable a la calidad de la organización del evento, siendo esta una posible explicación. No obstante, se deberá indagar más para determinar por qué en este caso la accesibilidad obtuvo bajos índices de fiabilidad y validez.

Resulta sorprendente cómo la calidad en global tiene un efecto mayor sobre el coste percibido que sobre la satisfacción. Se consigue explicar un $66,4 \%$ de la varianza de la satisfacción, lo cual indica que las variables predictoras de la satisfacción: la calidad y el valor, han sido bien elegidas. En este caso, es destacable que el mayor peso lo ejerce el valor coste percibido configurándose a partir de este hallazgo en una variable a tener muy en cuenta en la gestión de los eventos deportivos. Resultados similares aportan Calabuig et al. (2010a), dónde 
es el valor percibido el que consigue explicar en mayor medida la satisfacción de los espectadores de eventos de atletismo. En cambio Clemes et al. (2011) encuentran el efecto contrario, siendo la calidad la que mayor peso ejerce en explicar la satisfacción en oposición al valor.

También se coincide con Theodorakis et al. (2001) respecto al papel de los elementos tangibles del evento. Se conforma como la variable de mayor peso en la explicación del valor coste percibido y de la satisfacción del espectador. También resulta sorprendente que el personal del evento tenga más peso en la predicción, tanto de la satisfacción como del valor coste, que los servicios complementarios. Es de esperar que en un evento deportivo donde el contacto con los empleados es mínimo, su influencia sea menor que por ejemplo las cafeterías, aseos u otras concesiones.

En definitiva, respecto al primer objetivo de la investigación, los datos sugieren que los elementos tangibles y el valor monetario percibido por los espectadores de un gran evento deportivo son determinantes para explicar la satisfacción general de los espectadores. Además, se observa cómo el valor monetario ejerce, por sí solo, un fuerte efecto sobre la satisfacción debiéndose tener muy en cuenta por parte de los gestores de estos eventos si quieren mejorar el nivel de satisfacción de los espectadores. Estos resultados coinciden en parte con los de Theodorakis et al. (2001) pues observan el mismo efecto de los tangibles sobre la satisfacción de los espectadores aunque no se coincide respecto al papel de la accesibilidad y el personal. También se coincide con Kim et al. (2006), los cuales encuentran que la calidad es un fuerte predictor de la satisfacción, en cambio, no lo es tanto sobre las intenciones de volver a consumir.

En relación al segundo objetivo del trabajo, es decir a la importancia del valor coste o valor monetario sobre las intenciones futuras del espectador, cabe decir que los resultados son sorprendentes y abren nuevas vías de investigación para el futuro. Así, se observa en la Figura 2 como el valor coste es el que ejerce prácticamente todo el peso en la predicción de las intenciones futuras del espectador en comparación a la satisfacción general. Se consigue explicar el $67,4 \%$ de la varianza de las intenciones futuras y aunque la satisfacción también muestra una relación significativa, el peso es escaso. Tradicionalmente la satisfacción ha sido la variable más influyente en las intenciones de los usuarios, en cambio, a partir de estos resultados se deberá prestar más atención, tanto desde el mundo profesional como desde el académico, a aspectos relacionados con el coste percibido por los espectadores.

Así pues, los gestores de eventos deportivos deben hacer esfuerzos por mejorar la calidad de los tangibles y el valor monetario de sus servicios para aumentar la satisfacción de los espectadores y retenerles para el futuro. En definitiva y a modo de conclusión, el coste percibido pasa a ser un elemento central en la formación de la satisfacción y de las intenciones comportamentales 
de los espectadores de eventos deportivos y en consecuencia se deberán hacer más esfuerzos desde lo profesional, pero sobre todo desde el mundo académico, por intentar diseccionar el constructo de valor coste percibido y comprender mejor los mecanismos de su formación para mejorar, en definitiva, la rentabilidad de los eventos deportivos.

\section{REFERENCIAS BIBLIOGRÁFICAS}

ANDERSON JC, y GERBING DW. (1988). "Structural equation modelling in practice: a review and recommended two-step approach". Psychological Bulletin, 103(3), pp. 411-23.

ANDERSON, E.W.; FORNELL, C. y LEHMANN, D.R. (1994). "Customer satisfaction, market share, and profitability: Findings from Sweden". Journal of Marketing, 58(3), pp. 53-66.

BAGOZZI, R.P., y Yi, Y. (1988). "On the evaluation of structural equation models". Journal of the Academy of Marketing Science, 16, pp. 74-94.

BAKER, D. y CROMPTON, J.L. (2000). "Quality, satisfaction and behavioral intentions". Annals of Tourism Research, 27(3), pp. 785-804.

BENTLER, P.M. (1990). "Comparative fit indexes in structural models". Psychological Bulletin, 107, pp. 238-46.

BENTLER, P.M. (1995). EQS Structural Equations Program Manual, Encino, CA, Multivariate Software, Inc.

BODET, G. y BERNANCHE-ASSOLLANT, I. (2011). "Consumer loyalty in sport spectatorship services: The relatinship with consumer satisfaction and team identification". Psycology \& Marketing, 28(8), pp. (781-802).

BOJANIC, D.C. (1996). "Consumer Perceptions of Price, Value and Satisfaction in the Hotel Industry: An Exploratory Study". Journal of Hospitality and Leisure Marketing, 4(1), pp. 5-22.

BROWNE, M.W. y CUDECK, R. (1993). Alternative ways of assessing model fit. In: Bollen, K. A. \& Long, J. S. (Eds.) Testing Structural Equation Models. pp. 136-162. Beverly Hills, CA: Sage

BYRNE, B (1989). A primer of LISREL: Basic applications and programming for confirmatory factor analytic models. New York: Springer-Verlag.

CALABUIG, F.; BURILLO, P.; CRESPO, J.; MUNDINA, J. y GALLARDO, L. (2010a). "Satisfacción, calidad y valor percibido en espectadores de atletismo". Revista Internacional de Medicina y Ciencias de la Actividad Física y el Deporte, 10(40), 577-593.

CALABUIG, F.; MOLINOS, M. y SALA, R. [Eds.] (2012). Economía del deporte en tiempos de crisis. Valencia: Diazotec. 
CALABUIG, F.; MUNDINA, J. J.; y CRESPO, J. (2010b). "Eventqual: Una medida de la calidad percibida por los espectadores de eventos deportivos". Retos. Nuevas Tendencias en Educación Física, Deporte y Recreación, 18, pp. 66-70.

CARMINES, E.G. y McIVER, J.P (1981). Analyzing models with unobserved variables, en G.W. BOHRNSTEDT, y E.F. BORGATTA (Eds.), Social measurement: Current issues (pp. 65-115). Beverly Hills, CA: Sage.

CARUANA, A. y FENECH, N. (2005). "The effect of perceived value and overall satisfaction on loyalty: a study among dental patients". Journal of Medical Marketing, 5(3), pp. 245-255.

CHANG, H.; WNAG, Y. y YANGA, W. (2009). "The impact of e-service quality, customer satisfaction and loyalty on e-marketing. Moderating effect of perceived value". Total Quality Management, 20(4), pp. 423-443.

CHEN, Z. y DUBINSKY, A. J. (2003). "A conceptual model of perceived customer value in e-commerce: A preliminary investigation". Psychology \& Marketing, 20(4), pp. 323-347.

CLEMES, M.D.; BRUSH, G.J. y COLLINS, M.J. (2011). "Analysing the professional sport experience: A hierarchical approach". Sport Management Review, 14(4), pp. 370-388.

CRONIN, J.J. y TAYLOR, S.A. (1992). "Measuring service quality: A reexamination and extension". Journal of Marketing, 56(3), 55-68.

CRONIN, J.J.; BRADY, M.K. y HULT, G.T. (2000). "Assessing the effects of quality, value and customer satisfaction on consumer behavioral intentions in service environments". Journal of Retailing, 76(2), 193-218.

DODDS, W. B.; MONROE, K. B. y GREWAL, D. (1991). "The effects of price, brand and store information on buyers' product evaluations". Journal of Marketing Research, 28(3), pp. 307-319.

FORNELL, C. (1992). "A national customer satisfaction barometer: The Swedish experience" en Journal of Marketing, 56(1), pp. 6-21.

FORNELL, C., y LARCKER, D. F. (1981). "Evaluating structural equations models with unobservable variables and measurement error". Journal of Marketing Research, (18), pp. 39-50.

FORNELL, C.; MICHAEL D.; JOHNSON, E. W.; ANDERSON, J. y BARBARA E. (1996). "The American Customer Satisfaction Index: Nature, purpose, and findings". Journal of Marketing, 60(4), pp. 7-18.

GALLARZA, M. y GIL, I. (2006). "Value dimensions, perceived value, satisfaction and loyalty: an investigation of university students' travel behavior". Tourism Management, 27(3), pp. 437-452.

GÓMEZ, A.M. y PALACÍ, F.J. (2003). "El constructo orientación a la calidad total y su medida: el cuestionario OCT". Psicothema, 15(1), pp. 433-439.

GREENWELL, T.C.; FINK, J.S. y PASTORE, D.L. (2002). "Assessing the influence of the physical sports facility on customer satisfaction within the context of service experience". Sport Management Review, 5(2), 129-148. 
HAIR, J.F.; BLACK, W.C.; BABIN, B.J.; ANDERSON, R.E., y TATHAM, R.L. (2006). Multivariate Data Analysis (6th ed). New York: MacMillion Publishing Company.

HIGHTOWER, R.; BRADY, M.K. y BAKER, T.L. (2002). "Investigating the role of the physical environment in hedonic service consumption: an exploratory study of sporting events". Journal of Business Research, 55(9), 697-707.

HU, L., y BENTLER, P.M. (1999). "Cutoff criteria for fit indexes in covariance structure analysis: Conventional criteria versus new alternatives". Structural Equation Modeling: A Multidisciplinary Journal, 6(1), pp. 1-55.

HUME, M. (2008). "Understanding core and peripheral service quality in customer repurchase of the performing arts". Managing Service Quality, 18(4), pp. 349-369.

JAYANTI, R. K. y GHOSH A.K. (1996). "Service Value Determination: An Integrative Perspective". Journal of Hospitality and Leisure Marketing, 34(4), pp. 5-25.

JÖRESKOG, K.G., y SÖRBOM, D. (2001). LISREL 8.50. Chicago: Scientific Software International.

KAPLAN, D. (2000). Structural Equation Modeling: Foundations and Extensions. California, Thousand Oaks: Sage Publications.

KELLEY, S.W. y TURLEY L.W. (2001). "Consumer perceptions of service quality attributes at sporting events". Journal of Business Research, 54(2), 161-166.

KIM, H.; LAVETTER, D., y LEE. J. (2006). "The influence of service quality factors on customer satisfaction and repurchase intention in the Korean professional basketball league". International Journal of Applied Sports Sciences, 18(1), pp. 39-58.

KO, Y.; ZHANG, J.; CATTANI, K. y PASTORE, D. (2011) "Assessment of event quality in major spectator sports". Managing Service Quality, 21(3), pp. 304322.

KWON, H.H.; TRAIL, G.T. y ANDERSON, D. (2005). "Are points of attachement necessary in predicting cognitive, affective, concative, or behavioral loyalty? A case analysis". Sport Management Review, 8(3), pp. 255-270.

LAVERIE, D.A., y ARNETT, D.B. (2000). "Factors affecting fan attendance: The influence of identity salience and satisfaction". Journal of Leisure Research, 32(2), pp. 225-246.

LUQUE-MARTÍNEZ, T. [coord.] (2004). Técnicas de análisis de datos en investigación de mercados. Madrid: Pirámide.

MACCALLUM, R.C. y AUSTIN, J.T (2000). "Applications of structural equation modeling in psychological research". Annual Review of Psychology, 51, pp. 201-226.

NUNNALLY, J. C., y BERNSTEIN, I. H. (1994). Psychometric theory (3erd ed.). New York: McGraw Hill. 
OLIVER, R.L. (1997). Satisfaction: A behavioral perspective on the consmer. New York: McGraw-Hill.

OLIVER, R.L.; RUST, R.T.; y VARKI, S. (1997). "Customer delight: foundations, findings, and managerial insight". Journal of Retailing, 73, pp. 311-336.

PARASURAMAN, A.; ZEITHAML, V. A. y BERRY, L. L. (1988). "SERVQUAL: A multi-item scale for measuring consumer perceptions of service quality". Journal of Retailing, 64(1), pp. 12-40.

PRICE, L.L., y ARNOULD, E.J. (1999). "Commercial friendships: Service provider-client relationships in context". Journal of Marketing, 63(4), pp. 38-56.

SÁNCHEZ, M. (1996). "A strategy for the assessment of overall model fit in structural equation modelling for business economics" en Estudios de Economía Aplicada, 6, pp. 183-210.

SÁNCHEZ, R. y INIESTA, M.A. (2006). "Consumer perception of value: Literature Review and a New Conceptual Framework". Journal of Consumer Satisfaction, Dissatisfaction and Complaining Behavior, 19, pp. 40-59.

SETIJONO, D. y DAHLGAARD, J.J. (2007). "Customer value as a key performance indicator (KPI) and a key improvement indicator (KII)". Measuring Business Excellence, 11(2), pp. 44-61.

SHONK, D.J. y CHELLADURAI, P. (2008). "Service quality, satisfaction, and intent to return in event sport tourism". Journal of Sport Management, 22(5), pp. 587-602.

STEIGER, J.H. (1990). "Structural model evaluation and modification: an interval estimation approach". Multivariate Behavior Research, 25, pp. 173-80.

SZYMANSKI, D.M. y HENARD, D.H. (2001). "Customer Satisfaction: A MetaAnalysis of the Empirical Evidence". Journal of the Academy of Marketing Science, 29(1), pp. 16-35.

THEODORAKIS, N. y ALEXANDRIS, K. (2008). "Can service quality predict spectators' behavioral intentions in professional soccer?". Managing Leisure, 13(3), pp. 162-178.

THEODORAKIS, N.; KAMBITSIS, C. y LAIOS, A. (2001). "Relationship between measures of service quality and satisfaction of spectators in professional sports". Managing Service Quality, 11(6), pp. 431-438.

TRAIL, G.T.; ANDERSON, D.F. y FINK, J.S. (2005). "Consumer satisfaction and identity theory: A model of sport spectator conative loyalty". Sport Marketing Quarterly, 14(2), pp. 98-112.

TSITSKARI, E.; TSIOTRAS, D. y TSIOTRAS, G. (2006): "Measuring service quality in sport services". Total Quality Management \& Business Excellence, 17(5), pp. 623-631.

TSUJI, Y.; BENNETT, G. y ZHANG, J. (2007). "Consumer satisfaction with an action sports event”. Sport Marketing Quarterly, 16, pp. 199-208. 
VANDENBOSCH, M.B. (1996). "Confirmatory compositional approaches to the development of product spaces". European Journal of Marketing, 30(3), pp. 23-46.

WAKEFIELD, K.L. y BLODGETT, J.G. (1996). "The effect of the servicescape on customers' behavioral intentions in leisure service settings" en Journal of Services Marketing, 10(6), pp. 45-61.

WANGENHEIM, F., y BAYÓN, T. (2007). "The chain from customer satisfaction via word-of-mouth referrals to new customer acquisition". Academy of Marketing Science Journal, 35(2), pp.233-249.

ZEITHAML, V.A. (1988). "Consumer Perceptions of Price, Quality, and Value: A Means-End Model and Synthesis of Evidence". Journal of Marketing, 52(3), pp. 2-22.

ZEITHAML, V.A.; BERRY, L.L. y PARASURAMAN, A. (1996). "The behavioral consequences of service quality" en Journal of Marketing, 60, pp. 31-46. 


\section{ANEXO}

Tabla 3

Cuestionario utilizado en la investigación con indicación de constructos y dimensiones

\section{Calidad Percibida}

Dimensión Accesibilidad

Ha resultado sencillo adquirir mi entrada *

He conseguido la entrada que deseaba *

He localizado mi asiento con facilidad *

Dimensión Personal

Hay suficiente personal de la organización para atender a los usuarios

Los empleados de la organización están bien formados

Los empleados de la organización realizan bien su trabajo

Los empleados de la organización atienden con amabilidad

Dimensión Tangibles

La visión del evento es buena

En general, la audición es correcta

En general, la limpieza de las instalaciones es correcta

Al entrar a la instalación me he sentido impresionado

La instalación me produce buenas sensaciones

La instalación es ideal para la práctica deportiva

Se da buena información para conocer el desarrollo del evento *

Dimensión Servicios Complementarios

El servicio de cafetería ha cumplido con mis necesidades

Los empleados del servicio de cafetería realiza bien su trabajo

La limpieza e higiene de los aseos es correcta

Existe una buena oferta de productos del evento (cantidad, calidad, precio,...) *

\section{Satisfacción general}

Estoy contento con las experiencias que he tenido en el evento

Estoy satisfecho con mis experiencias en este evento

Verdaderamente he disfrutado asistiendo a evento

Estoy emocionado con las experiencias que he tenido en este evento

\section{Valor coste percibido}

En general, asistir al evento compensa lo pagado

$\mathrm{Al}$ asistir al evento, he recibido mucho por el dinero que he pagado

\section{Intenciones futuras}

Estaría dispuesto a continuar asistiendo a este evento

Recomendaré la asistencia a mis amigos y parientes

Si tengo la oportunidad de volver a asistir, repetiré la experiencia

Nota: * indica que el ítem fue eliminado en el proceso de validación.

Fuente: Elaboración propia. 